\title{
Daily anticipatory rhythms of behavior and body temperature in response to glucose availability in rats
}

\author{
Breno T.S. Carneiro, Diego A.C. Fernandes, Caio F.P. Medeiros, Nathália L. Diniz, John F. \\ Araujo \\ Universidade Federal do Rio Grande do Norte, Natal, RN, Brazil.
}

\begin{abstract}
When food is available recurrently at a particular time of day, several species increase their locomotion in the hours that precede food delivery, a phenomenon called food anticipatory activity (FAA). In mammals, many studies have shown that FAA is driven by a food-entrained circadian oscillator (FEO) that is distinct from the light-entrained pacemaker in the suprachiasmatic nucleus of the hypothalamus. Few studies have investigated the effect of sugar ingestion on food anticipatory rhythms and the FEO. We aimed to extend the understanding of the role of glucose on the emergence of food anticipatory rhythms by investigating whether glucose ingestion is sufficient to produce daily food anticipation, reflected by motor activity and core body temperature rhythms. Under a $12 \mathrm{~h} / 12 \mathrm{~h}$ light/dark cycle, chow-deprived rats had glucose solution available between Zeitgeber Time (ZT) 6 and ZT 9 for 10 days (glucose restriction group), whereas control animals had chow available within the same time window (chow restriction group). Animals in both groups exhibited anticipatory motor activity and body temperature around the fourth day of the scheduled food restriction. Glucose-fed rats ingested $\sim 15 \mathrm{kcal}$ on the days immediately before FAA emergence and reached an intake of $\sim 20 \mathrm{kcal} /$ day, whereas chow-fed rats ingested $\sim 40 \mathrm{kcal} /$ day. The glucose restriction group exhibited a pattern of food anticipation (activity and temperature) that was extremely similar to that observed in the chow restriction group. We conclude that glucose ingestion is a sufficient temporal cue to produce recurrent food anticipation, reflected by activity and temperature rhythms, in rats. Keywords: food-entrained oscillator (FEO); scheduled food restriction; food anticipatory rhythms; glucose
\end{abstract}

Received 27 August 2012; received in revised form 04 December 2012; accepted 05 December 2012. Available online 28 December 2012.

\section{Introduction}

Circadian rhythms are $\sim 24 \mathrm{~h}$ oscillations present in several species. They include biochemical, physiological, behavioral, and cognitive rhythms in organisms as diverse as bacteria and mammals (Campos, Silva, Fernandes, Araujo, \& Menezes, 2001; DeCoursey, 2004). Circadian rhythms entrain (i.e., synchronize) to cyclic environmental cues (zeitgebers, from the German for "time-givers"), of which the most evident is the daily light/ dark cycle created by the earth's rotation. In mammals, other cues are important for organizing behavioral and physiological rhythms. One of them is food availability, which is perhaps the most restrictive zeitgeber because it directly involves the animal's survival. Rats and mice exhibit food anticipatory activity (FAA) when food

Breno T.S. Carneiro, Diego A.C. Fernandes, Caio F.P. Medeiros, Nathália L. Diniz, and John F. Araujo, Departamento de Fisiologia, Centro de Biociências, Universidade Federal do Rio Grande do Norte, Natal, RN, Brazil. Breno T.S. Carneiro, Diego. Correspondence regarding this article should be directed to Breno T.S. Carneiro, Universidade Federal Rural do Semi-Árido (UFERSA), Departamento de Ciências Animais, Campus Mossoró - Oeste, Av. Francisco Mota, 572, Presidente Costa e Silva, Mossoró, RN, CEP 59625-900, Brazil. E-mail: brenocarneiro@ufersa.edu.br availability is restricted to a particular time each day (scheduled food restriction; Mistlberger, 1994). FAA is characterized by an increase in locomotion in the hours that precede food availability. Much evidence indicates that FAA is the manifestation of a food-entrained oscillator (FEO), which exhibits circadian properties (Mistlberger, 1994; Stephan, 2002). FAA emerges only when the cycle of food availability (i.e., its period) occurs in a range of $23-29 \mathrm{~h}$.

Considering the importance of food ingestion for the proper functioning of the organism, food anticipatory rhythms have been observed in different species (Mistlberger, 1994; Ware, Nelson, Robbins, \& Jansen, 2012) and probably evolved because of the advantage that it confers for survival. Recent molecular tools and genetic manipulations in rodents have allowed the search and identification of food-entrained clocks in the whole body including the central nervous system and peripheral tissues (e.g., liver, stomach, and intestines; Stokkan, Yamazaki, Tei, Sakaki, \& Menaker, 2001; Davidson, Poole, Yamazaki, \& Menaker, 2003; Verwey \& Amir, 2009; Mendoza, Pévet, Felder-Schmittbuhl, Bailly, \& Challet, 2010). The discovery of these food-entrained clocks in the whole body has led to propositions that food anticipatory rhythms are controlled by interactions between peripheral and central food-entrained clocks 
(Escobar, Cailotto, Angeles-Castellanos, Delgado, \& Buijs, 2009). However, despite intense research, the location and organization of the FEO and its entraining pathways and mechanisms have not been described (Davidson, 2009; Carneiro \& Araujo, 2009). The finding that sympathetic and parasympathetic afferents are unnecessary for the emergence of FAA in rats has led to the notion that a circulating humoral factor may be involved in the gut-brain communication that is thought to be necessary for the expression of food anticipatory behaviors (Comperatore \& Stephan, 1990; Davidson \& Stephan, 1998).

Few studies have investigated whether specific macronutrients play differential roles in FAA (Mistlberger, Houpt, \& Moore-Ede, 1990; Stephan \& Davidson, 1998; Pecoraro, Gomez, Laugero, \& Dallman, 2002). Studies on this subject may suggest the mechanisms by which behavioral and physiological parameters are entrained to food availability. Few studies have investigated the effect of sugar ingestion on food anticipatory rhythms. Pecoraro et al. (2002) tested the effect of timed sucrose ingestion at $4 \%$ and $32 \%$ in partially food-deprived rats and reported that it elicited anticipatory rhythms of activity and body temperature. Employing shifts of the feeding time, Stephan \& Davidson (1998) reported that rats that ingested glucose during the first 2 days of a phaseshifted feeding time exhibited faster FAA readjustment than rats that received saccharin, vegetable, or mineral oil. This observation indicates that glucose per se can interfere with the FEO, but these results do not indicate whether glucose ingestion itself is sufficient to produce recurrent daily FAA. Here we sought to extend the understanding of the role of glucose in the emergence of food anticipatory rhythms by investigating whether glucose ingestion is sufficient to produce daily FAA. We recorded motor activity and core temperature rhythms in completely chow-deprived rats subjected to scheduled glucose availability.

\section{Material and methods Subjects}

Forty-eight (36 males, 12 females) Wistar rats (2-4 months of age) were used in the study. Animals were individually housed in polypropylene cages (40 $\times 32 \times 17 \mathrm{~cm}$ ) inside light-tight, ventilated wooden cabinets under a controlled light/dark cycle (100-280 lux in the light phase; $<1$ lux in the dark phase) and temperature $\left(23 \pm 1^{\circ} \mathrm{C}\right)$ with tap water and food (rodent chow, Labina, Purina; $3.5 \mathrm{kcal} / \mathrm{g}$ ) provided ad libitum except during scheduled food restriction. All protocols were approved by the ethics commission on the use of animals of the Federal University of Rio Grande do Norte (project no. 026/2009). The study was performed in compliance with the ethical principles of the Brazilian Society of Sciences of Laboratory Animals (SBCAL).

\section{Apparatus}

A subgroup of animals $(n=11)$ was anesthetized with i.p. injections of ketamine $(100 \mathrm{mg} / \mathrm{kg})$ and xylazine $(10 \mathrm{mg} / \mathrm{kg}$; União Química Farmacêutica Nacional S/A, São Paulo, SP, Brazil) and implanted with iButton temperature sensors (Maxim Integrated Products, Sunnyvale, CA, USA). The sensors were inserted through a small incision $(\sim 2 \mathrm{~cm})$ into the peritoneal cavity. The rats were allowed to recover for at least 14 days before beginning the experiment. The same surgical procedures were used to remove the sensors after the end of the experiment.

Motor activity was continuously detected in all animals using infrared motion sensors placed $15 \mathrm{~cm}$ above the cage lids. Data were collected using Aschoff software designed in our laboratory and stored on a computer at 15-min intervals. Temperature sensors were set to collect data every $30 \mathrm{~min}$ and the recordings were transferred to a computer after the end of the experiment. Actograms and thermograms were drawn using El temps software (A. Diez-Noguera, Universitat de Barcelona, 1999).

Glucose used in the study was provided in $50 \%$ concentration solutions inside plastic 10-ml flasks (2.0 kcal/ml, Isofarma, Industrial Farmacêutica Ltda, Eusébio, CE, Brazil).

\section{Procedure}

Motor activity was first recorded in 36 rats (24 males, 12 females). Animals were divided into three groups (ad libitum, glucose restriction [GR], and chow restriction [CR]; $n=12$ [eight males, four females] per group) and entrained to a $12 \mathrm{~h} / 12 \mathrm{~h}$ light/dark cycle for 10 days with food provided ad libitum (baseline) followed by 10 days of 3-h scheduled food restriction (glucose or chow) between Zeitgeber Time (ZT) 6 and 9. Subsequently, activity and temperature rhythms were recorded in a subgroup of 12 male rats ( $n=4$ per group) that was subjected to the protocol described above (temperature sensors were implanted in only three rats in the CR group). All animals were weighed 2-6 days before the beginning of the experiment (initial body weight) and on the last day of the experiment (day 20; final body weight). To allow the animals to learn to drink from the glucose flasks, each rat in the GR group received one flask at ZT 11.5 on days 7,8 , and 9. The flasks remained in the animals' cages for $24 \mathrm{~h}$. At the same time, the ad libitum group received flasks filled with tap water. On day 10, the food was removed from the GR and CR groups at ZT 11.5. During the food restriction days (starting on day 11), the GR and ad libitum groups received $20 \mathrm{ml}$ of glucose and tap water, respectively. Ingestion of glucose and chow was measured daily in the GR and CR groups, respectively. For glucose, we compared the remaining volume (if any) in the flasks with a flask with volume marks (.5 $\mathrm{ml}$ precision). The marks on the standard flask were determined previously with 10-ml syringes. For the CR group, we weighed the 
chow before and after scheduled feeding on a scale with 1 -g precision.

\section{Statistical analysis}

Motor activity, ingestion, and body weight data were combined from all 48 animals. Motor activity was transformed into a percentage of the daily total locomotion. FAA was defined as the total locomotion between ZT 4 and 6 and calculated for each day of the experiment. Total motor activity within this time window and activity and body temperature waveforms were averaged over days 1-5 (baseline) and days 16-20 (food restriction).

Caloric ingestion was compared between GR and $\mathrm{CR}$ groups using Student $t$-test for independent samples. Daily FAA, average activity between ZT 4 and 6 on baseline days and food restriction days, nocturnal activity, and body weight were compared among groups using one-way analysis of variance (ANOVA) followed by the Tukey post hoc test. The average activity between ZT 4 and 6 and body weight were also compared within groups using Student $t$-test for dependent samples. Body temperature waveforms were analyzed by visual inspection. Data are expressed as mean \pm SEM.

\section{Results}

Animals under glucose and chow restriction conditions exhibited increased motor activity and temperature in the hours that preceded food availability.
Food anticipation in both groups began to develop between the third and fourth days of scheduled food restriction. Anticipatory motor activity and body temperature are shown in the actograms and thermograms (Figure 1).

GR and CR groups changed their daily profiles of motor activity and temperature in response to scheduled food restriction. During baseline days, GR and $\mathrm{CR}$ groups exhibited both rhythms entrained to the light/dark cycle with low levels of locomotion and lower temperatures during the light phase. When foodrestricted, both groups showed increased locomotion and body temperature before mealtime (i.e., entrained food anticipatory rhythms; Figure 2).

Activity during the dark phase decreased in the $\mathrm{CR}$ and GR groups from $\sim 9000$ counts during baseline days to $\sim 3000$ counts during food restriction days (Figure $3 \mathrm{~A}$ ), whereas FAA increased (Figure 3B). Additionally, activity between ZT 4 and 6 on days 16-20 was significantly greater than activity on days $1-5$ in both CR and GR groups (CR: $8.35 \pm .9 \%$ vs. $2.94 \pm .41 \%$ of daily activity; GR: $8.76 \pm .72 \% v s .2 .84 \pm .56 \%$ of daily activity), indicating the development of FAA. No significant difference was found between the $\mathrm{CR}$ and GR groups (Figure 3C).

As expected, ingestion increased during scheduled food restriction (Figure 4). Caloric intake differed between groups on all days of food restriction. The GR group ingested $\sim 20 \mathrm{kcal} /$ day, whereas the $\mathrm{CR}$ group ingested $\sim 40 \mathrm{kcal} /$ day (Figure $4 \mathrm{~B}$ ). Body weight,
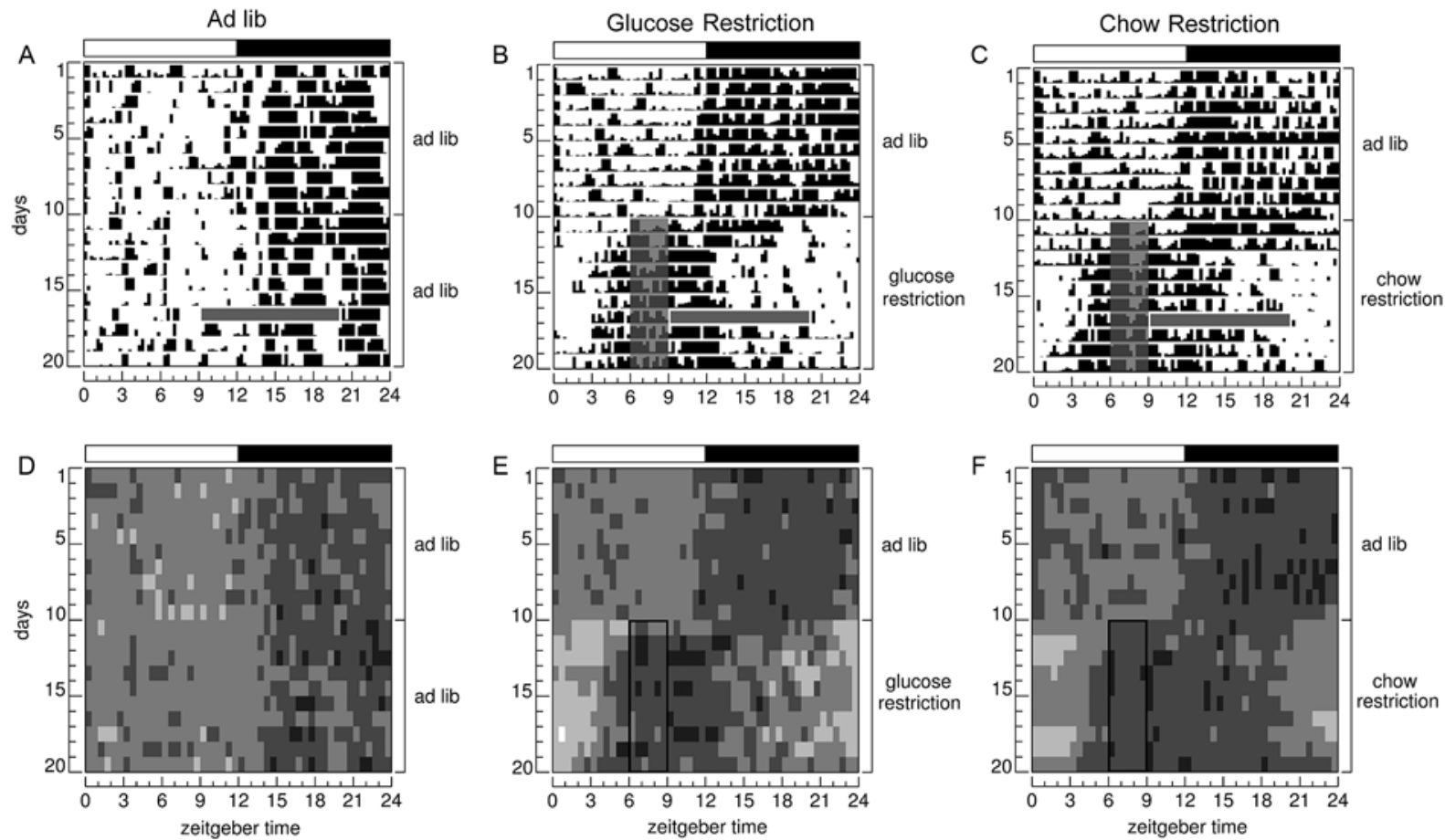

Figure 1. Circadian rhythms of motor activity and body temperature. Representative actograms (A-C) and corresponding thermograms (D-F) illustrate food anticipation in the GR group (B,E) and CR group (C,F). Chow and glucose availability are indicated by gray areas and black empty rectangles on the actograms and thermograms, respectively. Motor activity is represented in 15 min bins. Thermograms are presented in gray scale from $34^{\circ} \mathrm{C}$ to $39^{\circ} \mathrm{C}$. Missing activity data are shown as a horizontal gray bar in the actograms. 

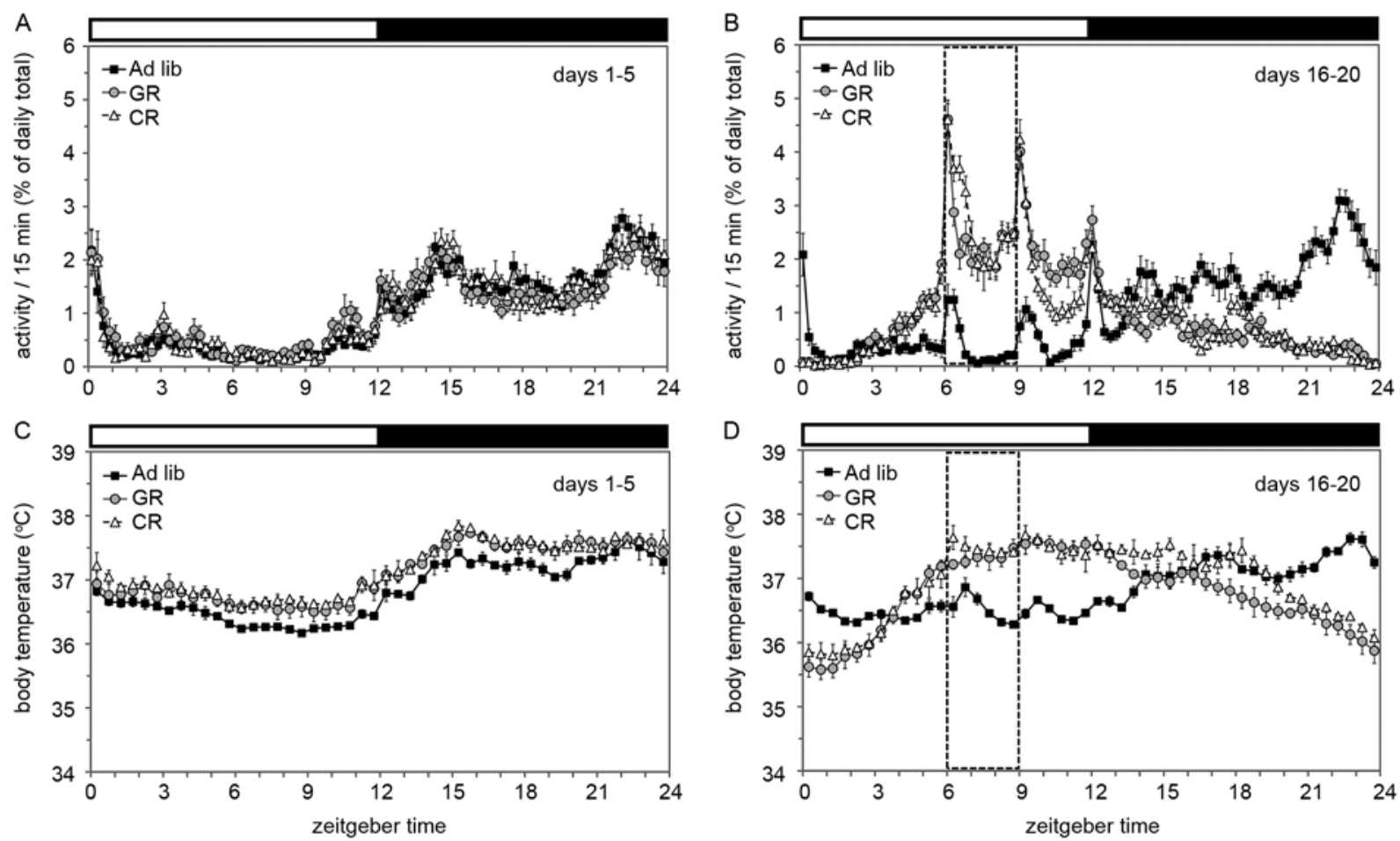

Figure 2. Activity and body temperature waveforms. (A,B) Activity waveforms averaged over days $1-5$ (baseline) and days 16-20 (food restriction). (C,D) Body temperature waveforms. Increases in locomotion and body temperature prior to food availability are clear in the GR and CR groups on the food restriction days.
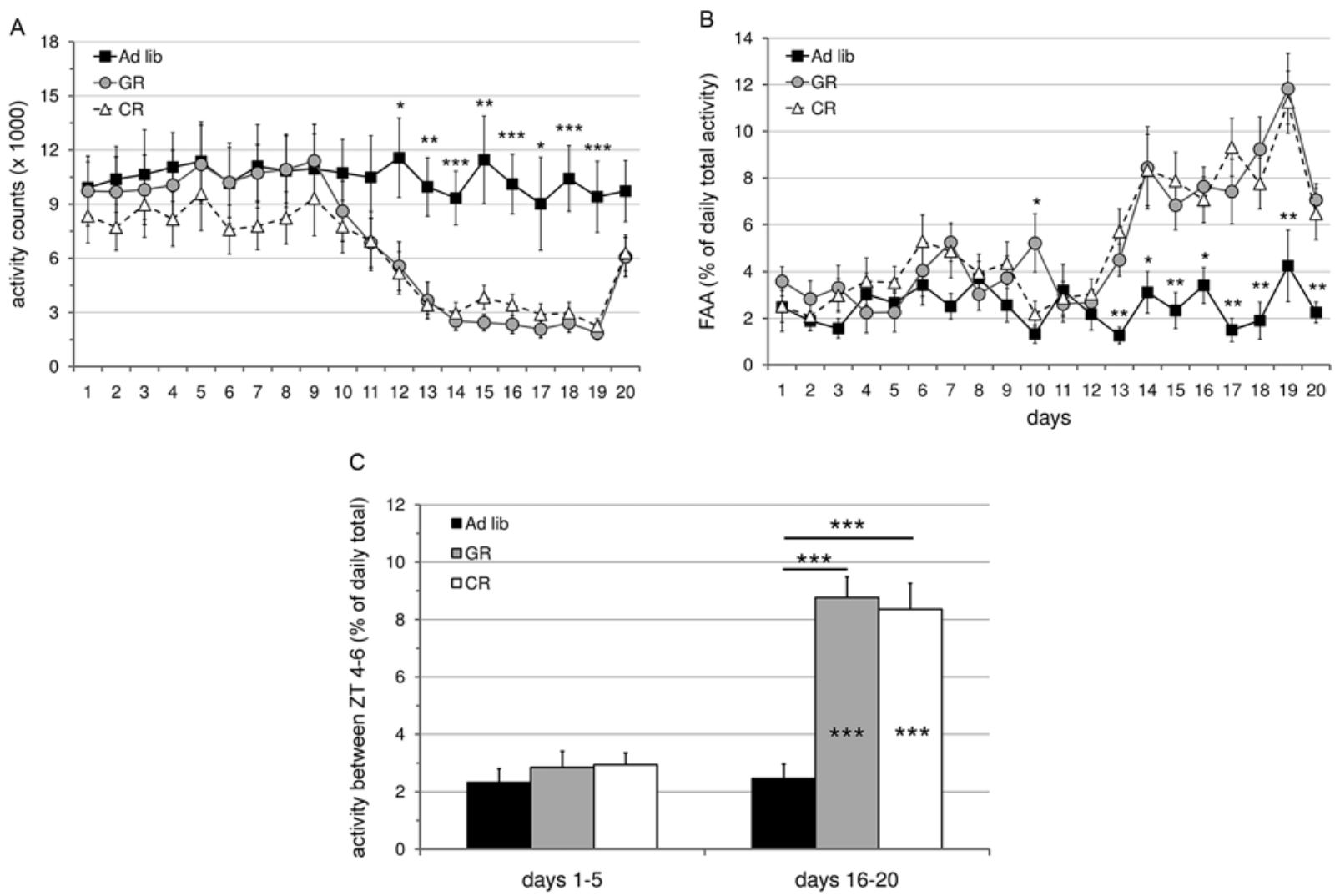

Figure 3. Food anticipatory and nocturnal activity. (A) Nocturnal activity decreased during the food restriction days (asterisks indicate differences between the ad libitum group and the GR and CR groups). (B) Food anticipatory activity along the food restriction days (asterisks indicate differences between the ad libitum group and the GR and CR groups). (C) Average motor activity between ZT 4 and 6 on days 1-5 (baseline) and days 16-20 (food restriction). Asterisks inside the columns indicate comparisons within groups between two conditions (baseline days and food restriction days). Thick horizontal lines indicate comparisons between groups. ${ }^{*} p<.05, * * p<.01, * * * p<.001$. 

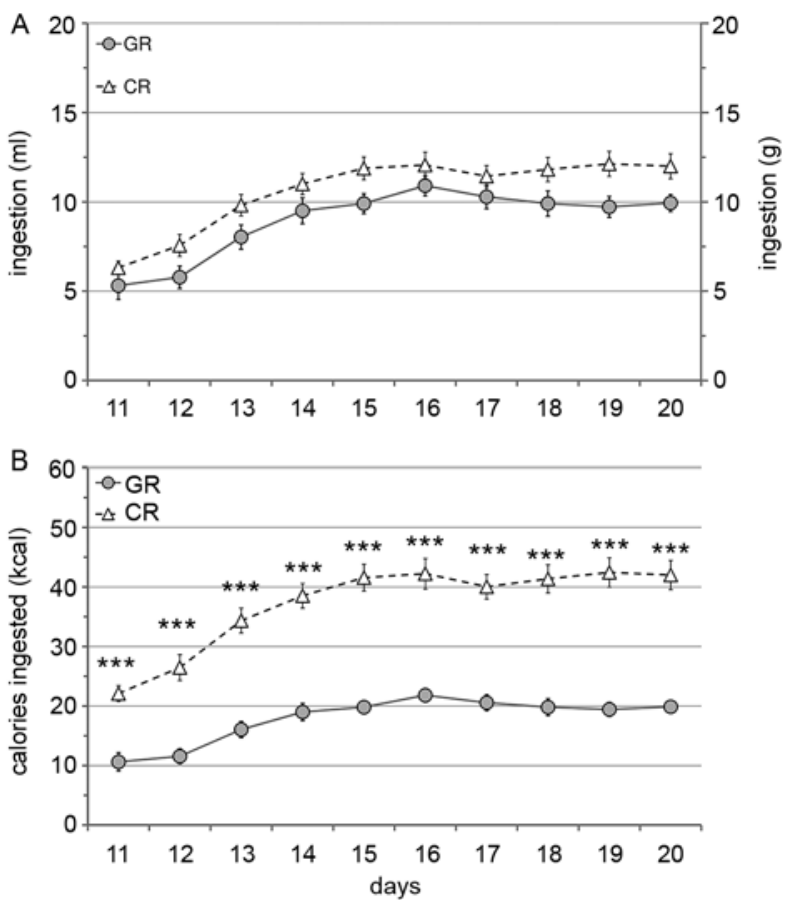

Figure 4. Ingestion during the food restriction days. (A) Ingestion in both groups in milliliters or grams. An increase was evident during the first 4 days. (B) Caloric ingestion, in which differences between groups were found on all days of food restriction. $* * * p<.001$.

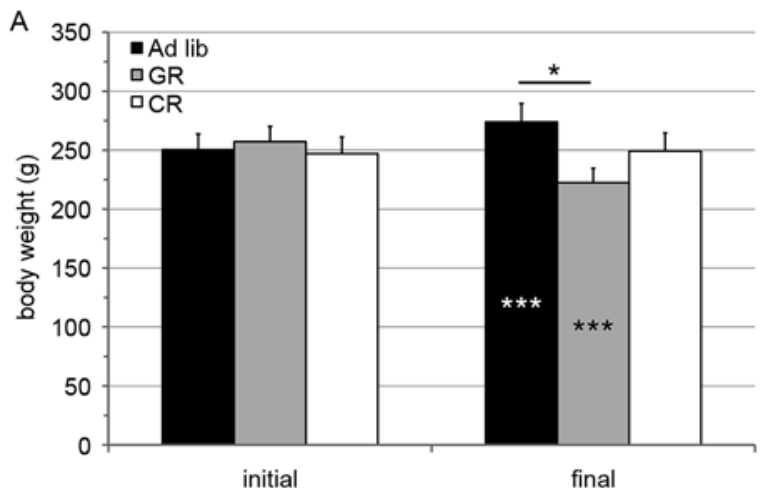

however, increased in the Ad libitum group, decreased in the GR group, and was unaffected in the CR group (Figure 5A and 5B). Additionally, individual body weight change plotted against FAA on days 16-20 revealed no correlation between FAA and weight loss (Figure 5C).

\section{Discussion}

In the present study we found that scheduled glucose ingestion was sufficient to induce anticipatory motor activity and body temperature in rats. In preliminary experiments without control groups, we studied only motor activity and observed persistent anticipation for glucose (Carneiro, Fortes, \& Araujo, 2010). In the present study, anticipatory motor activity and body temperature in rats under glucose restriction conditions were extremely similar to animals subjected to scheduled chow restriction. Both groups under scheduled food restriction showed a steep increase in locomotion in the $3 \mathrm{~h}$ that preceded food availability, going from almost zero activity to $2 \%$ of total daily activity in the 15 -min period immediately before food delivery (see Figure 2). For body temperature, a steep increase was also exhibited by both groups, with an increase of more than $1^{\circ} \mathrm{C}$ within the $3 \mathrm{~h}$ that preceded food availability (see Figure 2). These results indicate

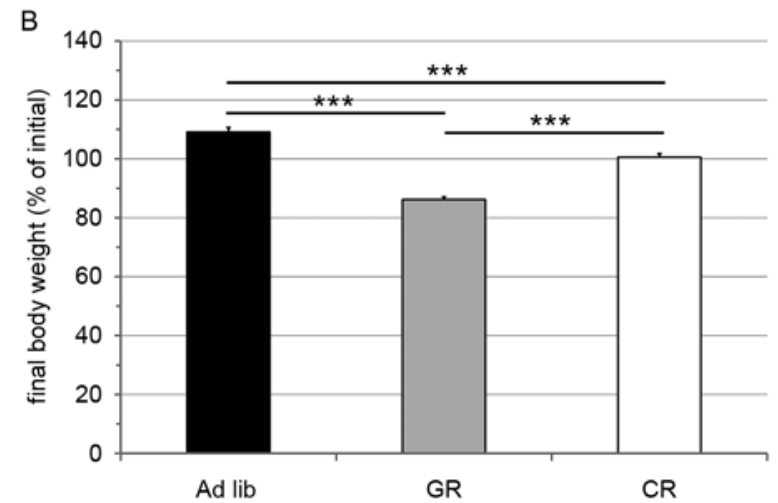

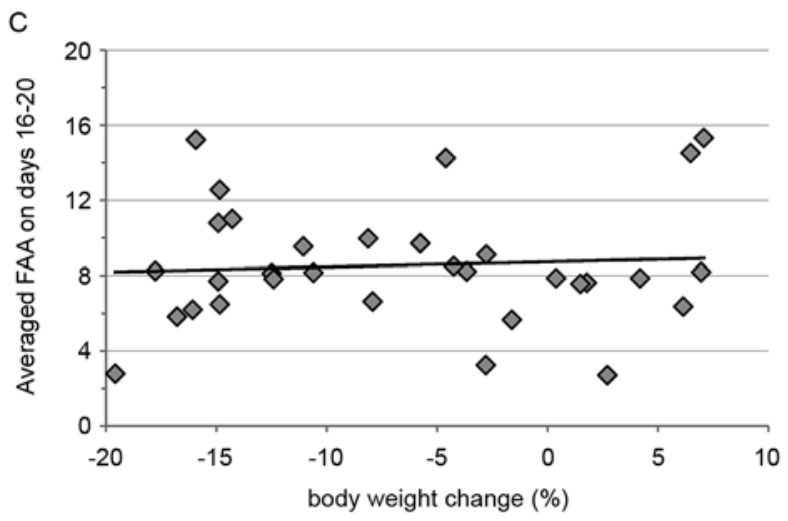

Figure 5. Body weight. (A) Initial and final body weight. (B) Final body weight as a percentage of initial weight. Asterisks within the columns indicate comparisons within groups between two conditions (initial and final body weight). (C) Correlation between weight change and FAA $(r=.0694, p>.71) .{ }^{* *} p<.01,{ }^{* * *} p<.001$. 
that anticipation in the animals that drank glucose was the same as FAA in the animals subjected to scheduled chow restriction.

Some evidence suggests dissociation between the neural control of behavior and physiology with regard to food entrainment, at least with regard to locomotion and temperature rhythms. Two reports showed that hypophysectomy or infralimbic cortical lesions abolished food anticipatory temperature but not locomotor rhythm in rats (Davidson \& Stephan, 1999; Recabarren, Valdés, Farías, Serón-Ferré, \& Torrealba, 2005). These results indicate the existence of two separate food-entrained brain subsystems, one that controls anticipatory locomotion and another that controls anticipatory body temperature. If these two separate systems indeed exist, then glucose ingestion is also sufficient to entrain both of them, given the anticipatory increase observed for locomotion and temperature rhythms in the present study.

As expected, both groups under scheduled food restriction conditions in the present study gradually increased ingestion. Nonetheless, because the chow used in the present study had greater caloric content than the glucose solution $(3.5 \mathrm{kcal} / \mathrm{g}$ vs. $2.0 \mathrm{kcal} / \mathrm{ml}$, respectively), the animals subjected to glucose restriction ingested fewer calories daily than the animals subjected to chow restriction. Stephan (1997) used a feeding time phase delay protocol to investigate the importance of the quantity of calories ingested on the expression of FAA in rats. The magnitude of the phase delays of anticipatory activity was influenced by the quantity of chow offered $(0,2,6$, or $16 \mathrm{~g})$ and ingestion during the first 2 days of the new feeding time. Furthermore, using the same protocol, Stephan (1997) confirmed the previous observations of Mistlberger \& Rusak (1987) in which caloric content and not gastric fill was critical for adjusting the FEO. The present results corroborate the previous results of Pecoraro et al. (2002) in which repeated timed sugar ingestion was sufficient to induce anticipatory rhythms in rats. That study reported that sucrose ingestion provided 15-20 kcal daily in animals that anticipated it. Stephan (1997) calculated that a minimum of $22 \mathrm{kcal}$ is necessary to entrain the FEO. Animals under glucose restriction conditions in the present study exhibited anticipatory activity that was visually evident between the third and fourth days of scheduled food restriction. On the days immediately prior (i.e., second and third), caloric ingestion was $\sim 15$ kcal. This value is similar to the values reported by Pecoraro et al. (2002).

The low quantity of calories ingested by animals under glucose restriction conditions was probably the cause for the greater weight loss observed in the GR group. Evidence indicates that body fat and the feeding-related hormones ghrelin and leptin modulate the intensity of FAA (Persons, Stephan, \& Bays, 1993; Mistlberger \& Marchant, 1999; Blum et al., 2009; Verhagen, Egecioglu, Luijendijk, Hillebrand, Adan, \& Dickson, 2011). Diet-induced adiposity and body weight are also associated with high leptin concentrations and low general ghrelin concentrations during fasting (Lindkvist, de La Cour, Stegmark, Hakanson, \& Erlanson-Albertsson, 2005). The contrary should be true for animals with low body weight and adiposity as in the present study in glucose-fed rats. In the present study, despite the greater weight loss in the GR group (see Figure 5B), we found no difference in FAA between the GR and CR groups and no correlation between weight loss and FAA levels (see Figure 5C), indicating that the probable modification of global leptin and ghrelin levels in our animals did not play any role in setting the intensity of FAA. Additionally, a recent report by Dailey, Stingl, \& Moran (2012) showed that rats on a chow meal schedule displayed almost twice the plasma ghrelin concentrations of animals on a high-fat meal schedule in the $1.5 \mathrm{~h}$ that preceded food availability, but their levels of food anticipatory behavior were similar. These results are consistent with two studies in which ghrelin and leptin individually did not play a significant role in physiological or behavioral food anticipation (Szentirmai, Kapás, Sun, Smith, \& Krueger, 2010; Gunapala, Gallardo, Hsu, \& Steele, 2011).

We used both male and female rats in this study. An earlier report showed that female rats prefer sweet solutions (e.g., glucose and saccharin) more than male rats (Valenstein, Kakolewski, \& Cox, 1967). Female rats in our study drank more glucose than male rats during the days before food restriction $(30 \mathrm{ml} v s .20 \mathrm{ml})$ when they learned to drink from the glucose flasks. However, we are unable to determine whether differential sweet preference in female rats affects anticipatory activity or temperature due to the small number of animals used in the present study.

We tested the effect of glucose restriction on activity and temperature rhythms specifically from ZT 6 to 9 . The time of day appears to modulate FAA intensity in rats, with nighttime scheduled food restriction producing more intense food anticipation than daytime scheduled food restriction (Landry, Kent, Patton, Jaholkowski, Marchant, \& Mistlberger, 2012). This result may be attributable to a differential behavioral effect (i.e., sleep or arousal promotion) exerted by the suprachiasmatic nucleus of the hypothalamus during the light and dark phases (Mistlberger, 2005), which possibly is achieved through brain areas that also control food anticipation. Such time-of-day modulation might also occur in anticipatory activity for glucose.

Feeding regimens that use carbohydrates have been shown to induce anticipation of feeder-directed activity in rats (Mistlberger et al., 1990). Ingestion of solid glucose produces phase shifts in the FEO (Stephan \& Davidson, 1998). This suggests that glucose may alter the activity of the FEO and is corroborated by the present results in which recurrent anticipatory behavioral and physiological rhythms were entrained by scheduled glucose ingestion. The identification of signals that are able to entrain the FEO may be relevant for manipulating circadian rhythms when considering the potential of scheduled feeding 
to manage circadian alterations (Ángeles-Castellanos, Amaya, Salgado-Delgado, Buijs, \& Escobar, 2011; Carneiro \& Araujo, 2011).

In the present study we removed all other nutrients from the feeding regimen and demonstrated that scheduled glucose ingestion is a sufficient temporal cue for inducing behavioral and physiological food anticipation in rats.

\section{Acknowledgments}

The present study was supported by Conselho Nacional de Desenvolvimento Científico e Tecnológico (CNPq, Brasil) and Fundação de Apoio à Pesquisa do Estado do Rio Grande do Norte (FAPERN, Brasil). We thank Bruna del Vechio Koike and João Miguel Ribeiro for assistance with the surgical procedures and Dr. Miriam S.M.O. Costa and André L.B. de Pontes for their comments on the manuscript. We also thank the two anonymous reviewers for their relevant and helpful comments on the manuscript.

\section{References}

Ángeles-Castellanos, M., Amaya, J. M., Salgado-Delgado, R., Buijs, R. M., \& Escobar, C. (2011). Scheduled food hastens reentrainment more than melatonin does after a 6 -h phase advance of the light-dark cycle in rats. Journal of Biological Rhythms, 26, 324-334.

Blum, I. D., Patterson, Z., Khazall, R., Lamont, E. W., Sleeman, M. W., Horvarth, T. L., \& Abizaid, A. (2009). Reduced anticipatory locomotor responses to scheduled meals in ghrelin receptor deficient mice. Neuroscience, 164, 351-359.

Campos, T. F., Silva, S. B., Fernandes, M. G., Araujo, J. F., \& Menezes, A. A. L. (2001). Diurnal variation in a visual-motor coordination test in healthy humans. Biological Rhythm Research, 32, 255-262.

Carneiro, B. T. S., \& Araujo, J. F. (2009). The food-entrainable oscillator: A network of interconnected brain structures entrained by humoral signals? Chronobiology International, 26, 1273-1289.

Carneiro, B. T. S., \& Araujo, J. F. (2011). Influence of scheduled restricted feeding on reentrainment of motor activity rhythm after a 6-h light-dark advance in rats. Psychology \& Neuroscience, 4, 317-322.

Carneiro, B. T. S., Fortes, F. S., \& Araujo, J. F. (2010). Anticipatory behavioral rhythm to scheduled glucose availability in rats. Sleep Science, 3, 27-31.

Comperatore, C. A., \& Stephan, F. K. (1990). Effects of vagotomy on entrainment of activity rhythms to food access. Physiology and Behavior, 47, 671-678.

Dailey, M. J., Stingl, K. C., \& Moran, T. H. (2012). Disassociation between preprandial gut peptide release and food-anticipatory activity. Endocrinology, 153, 132-142.

Davidson, A. J. (2009). Lesion studies targeting food-anticipatory activity. European Journal of Neuroscience, 30, 1658-1664.

Davidson, A. J., Poole, A. S., Yamazaki, S., \& Menaker, M. (2003). Is the food-entrainable oscillator in the digestive system? Genes, Brain and Behavior, 2, 32-39.

Davidson, A. J., \& Stephan, F. K. (1998). Circadian food anticipation in capsaicin deafferented rats. Journal of Biological Rhythms, 13, 422-429.

Davidson, A. J., \& Stephan, F. K. (1999). Feeding-entrained circadian rhythms in hypophysectomized rats with suprachiasmatic nucleus lesions. American Journal of Physiology: Regulatory, Integrative and Comparative Physiology, 277, R1376-R1384.

DeCoursey, P. J. (2004). Overview of biological timing from unicells to humans. In J. C. Dunlap, J. J. Loros, \& P. J. DeCoursey (Eds.), Chronobiology: Biological timekeeping (pp. 3-24). Sunderland, MA: Sinauer.
Escobar, C., Cailotto, C., Angeles-Castellanos, M., Delgado, R. S., \& Buijs, R. M. (2009). Peripheral oscillators: The driving force for food-anticipatory activity. European Journal of Neuroscience, 30, 1665-1675.

Gunapala, K. M., Gallardo, C. M., Hsu, C. T., \& Steele, A. D. (2011). Single gene deletions of orexin, leptin, neuropeptide $\mathrm{Y}$, and ghrelin do not appreciably alter food anticipatory activity in mice. PLOS One, 6, e18377.

Landry, G. J., Kent, B. A., Patton, D. F., Jaholkowski, M., Marchant, E. G., \& Mistlberger, R. E. (2012). Evidence for time-of-day dependent effect of neurotoxic dorsomedial hypothalamic lesions on food anticipatory circadian rhythms in rats. PLOS One, 6, e24187.

Lindkvist, A., de La Cour, C. D., Stegmark, A., Hakanson, R., \& Erlanson-Albertsson, C. (2005). Overeating of palatable food is associated with blunted leptin and ghrelin responses. Regulatory Peptides, 130, 123-132.

Mendoza, J., Pévet, P., Felder-Schmittbuhl, M. P., Bailly, Y., \& Challet, E. (2010). The cerebellum harbors a circadian oscillator involved in food anticipation. Journal of Neuroscience, 30, 1894-1904.

Mistlberger, R. E. (1994). Circadian food-anticipatory activity: formal models and physiological mechanisms. Neuroscience and Biobehavioral Reviews, 18, 171-195.

Mistlberger, R. E. (2005). Circadian regulation of sleep in mammals: role of the suprachiasmatic nucleus. Brain Research Reviews, 49, 429-454.

Mistlberger, R. E., Houpt, T. A., \& Moore-Ede, M. C. (1990). Foodanticipatory rhythms under 24-hour schedules of limited access to single macronutrients. Journal of Biological Rhythms, 5, 35-46.

Mistlberger, R. E., \& Marchant, E. G. (1999). Enhanced foodanticipatory circadian rhythms in the genetically obese Zucker rat. Physiology and Behavior, 66, 329-335.

Mistlberger, R., \& Rusak, B. (1987). Palatable daily meals entrain anticipatory activity rhythms in free-feeding rats: Dependence on meal size and nutrient content. Physiology and Behavior, 41, 219-226.

Pecoraro, N., Gomez, F., Laugero, K., \& Dallman, M. F. (2002). Brief access to sucrose engages food-entrainable rhythms in fooddeprived rats. Behavioural Neuroscience, 116, 757-776.

Persons, J. E., Stephan, F. K., \& Bays, M. E. (1993). Diet-induced obesity attenuates anticipation of food access in rats. Physiology and Behavior, 54, 55-64.

Recabarren, M. P., Valdés, J. L., Farías, P., Serón-Ferré, M., \& Torrealba, F. (2005). Differential effects of infralimbic cortical lesions on temperature and locomotor activity responses to feeding in rats. Neuroscience, 134, 1413-1422.

Stephan, F. K. (1997). Calories affect zeitgeber properties of the feeding entrained circadian oscillator. Physiology and Behavior, 62, 995-1002.

Stephan, F. K. (2002). The "other" circadian system: food as a Zeitgeber. Journal of Biological Rhythms, 17, 284-292.

Stephan, F. K., \& Davidson, A. J. (1998). Glucose, but not fat, phase shifts the feeding-entrained circadian clock. Physiology and Behavior, 65, 277-288.

Stokkan, K., Yamazaki, S., Tei, H., Sakaki, Y., \& Menaker, M. (2001). Entrainment of the circadian clock in the liver by feeding. Science, 291, 490-493.

Szentirmai, E., Kapás, L., Sun, Y., Smith, R. G., \& Krueger, J. M. (2010). Restricted feeding-induced sleep, activity, and body temperature changes in normal and preproghrelin-deficient mice. American Journal of Physiology: Regulatory, Integrative and Comparative Physiology, 298, R467-R477.

Valenstein, E. S., Kakolewski, J. W., \& Cox, V. C. (1967). Sex differences in taste preference for glucose and saccharin solutions. Science, 156, 942-943.

Verhagen, L. A. W., Egecioglu, E., Luijendijk, M. C. M., Hillebrand, J. J. G., Adan, R. A. H., \& Dickson, S. L. (2011). Acute and chronic suppression of the central ghrelin signaling system reveals a role in food anticipatory activity. European Journal of Neuropsychopharmacology, 21, 384-392.

Verwey, M., \& Amir, S. (2009). Food-entrainable circadian oscillators in the brain. European Journal of Neuroscience, 30, 1650-1657.

Ware, J. V., Nelson, O. L., Robbins, C. T., \& Jansen, H. T. (2012). Temporal organization of activity in the brown bear (Ursus arctos): Roles of circadian rhythms, light, and food entrainment. American Journal of Physiology: Regulatory, Integrative and Comparative Physiology, 303, R890-R902. 
